\title{
An efficient optimisation method in groundwater resource management
}

\author{
JM Ndambuki ${ }^{1 *}$, FAO Otieno ${ }^{2}$, CBM Stroet $^{3}$, T Terlaky $^{4}$ and EJM Veling ${ }^{3}$ \\ ${ }^{1}$ Department of Civil \& Structural Engineering, Moi University, PO Box 3900, Eldoret, Kenya \\ ${ }^{2}$ Faculty of Engineering, Technikon, Pretoria, Private Bag X680 Pretoria 0001, South Africa \\ ${ }^{3}$ Delft University of Technology, The Netherlands \\ ${ }^{4}$ McMaster University, Canada
}

\begin{abstract}
Uncertainty in input parameters to groundwater flow problems has been recognised as an impediment to designing efficient groundwater management strategies. The most popular approach to tackling this problem has been through the Monte Carlo approach. However, this approach is generally too expensive in terms of computer time because of the number of scenarios required to ensure reliable statistics. Furthermore, solutions obtained through this approach are not necessarily robust. In this paper, it is shown how groundwater management problems, where input parameters are uncertain can be reformulated as second-order cone optimisation (SOCO) problems, which are efficiently solved by recently developed interior-point methods. Results for a real-world case application of a groundwater aquifer found in Kenya are presented.
\end{abstract}

Keywords: Groundwater management, uncertainty, second-order cone, Laikipia, Kenya

\section{Introduction}

Theories of groundwater flow are well developed and over the last three decades, several applications have been made to model groundwater flow systems. A large number of these works have been based on deterministic flow simulation, and over the last decade a considerable volume of literature has been written and theories developed in the field of stochastic subsurface hydrology. In reality, many real-world aquifers are characterised by a few measurement points, which are used to derive the aquifer characteristics. Traditionally, the few measurement points available have been used for zonation purposes after which the management problem is solved deterministically. However, recognising the fact that the material forming aquifers varies enormously spatially, it is not immediately clear how optimal management strategies designed deterministically perform in an environment of uncertainty. Having noted that the parameters of the earth material which dictate the water-flow conditions vary a great deal spatially, one may ask the questions: Does it make sense to model groundwater systems deterministically using the sparse data available? How do solutions based on such an approach perform in a real-world scenario?

Geohydrologists, have for some time now, used the Monte Carlo approach in an attempt to desensitise the optimal solutions, thus including some robustness within the optimisation problem. However, the Monte Carlo approach is generally CPU-intensive (because of the large number of scenarios which have to be considered to arrive at a relatively insensitive optimal solution), hence its main drawback. Recently, some researchers have applied the multi-stage optimisation approach (in particular a two-stage optimisation approach) with some promising results (Ndambuki et al., 2000a; Wagner et al., 1992; Mulvey et al., 1995; Mark et al., 1999).

* To whom all correspondence should be addressed.

西+254-321-43292; fax: +254-321-43292;

e-mail: jmndambuki@yahoo.co.uk

Received 30 April 2003; accepted in revised form 19 August 2003.
In this paper, we transform our stochastic groundwater quantity management problem into a second-order cone optimisation problem, which is then solved by some powerful interior-point method. We first present a general introduction to second-order cone optimisation (SOCO) problems followed by the formulation of a second-order cone groundwater quantity management problem. Subsequently, we present results for a real world case aquifer found in Laikipia District, Kenya.

Consider a linear optimisation problem (LOP) of the following form:

$\operatorname{minimize} c^{T} x$

subject to:

$$
\begin{aligned}
& a_{i}^{T} x \leq b_{i}, i=1, \ldots, m \\
& x \geq 0
\end{aligned}
$$

where:

$c, a_{i} \in R^{n} ; b_{i} \in R$ are the problem parameters; while

$x$ are the optimisation variables.

Assuming that all the problem parameters except $a_{i}$ are accurately known and that $a_{i}$ is uncertain but lying in ellipsoids $\mathrm{e}_{\mathrm{i}}$ defined as:

$$
a_{i} \in \varepsilon_{i}=\left\{\overline{a_{i}}+\mathbf{P}_{i} u_{i} \mid\left\|u_{i}\right\| \leq 1\right\}
$$

where:

$\mathbf{P}=\mathbf{P}^{\mathrm{T}}$ are $n \times n$ perturbation matrices;

$a_{i}$ overstrike are the nominal values and the norm of $u_{i}$ ensure convexity.

Then a robust solution of the optimisation problem given by Eqs. (1) to (3) is as follows:

minimize $c^{T} x$

subject to:

$$
\begin{aligned}
& a_{i}^{T} x \leq b_{i}, \forall a_{i} \in \varepsilon_{i}, i=1, \ldots, m \\
& x \geq 0
\end{aligned}
$$




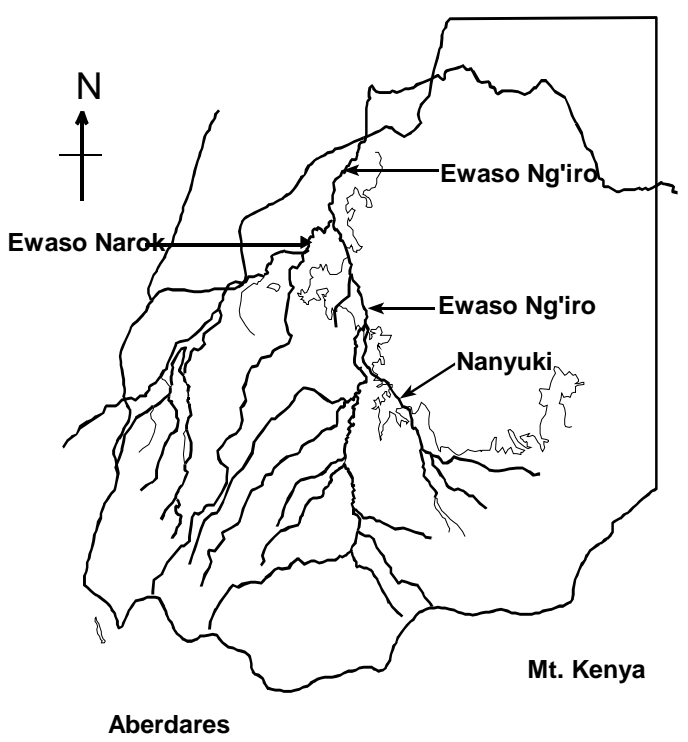

Figure 1

Study area

An optimisation problem defined by Eqs. (5) to (7), though now deterministic, has infinitely many constraints and a solution to this robust optimisation problem is feasible if for all $i=1, \ldots m$, the following holds (see e.g. Ben-Tal and Nemirovsky, 1998):

$$
\left.\mid \bar{a}_{i}^{T} x+\left(\mathrm{P}_{i} u_{i}\right)^{T} x-b_{i}\right\rfloor \leq 0, \forall u_{i}: u_{i}^{T} u_{i} \leq 1
$$

which can equivalently be reformulated by a single constraint as:

$$
{\overline{a_{i}}}^{T} x+\left\|\mathrm{P}_{i} x\right\| \leq b_{i}
$$

Constraints of the form Eq. (9) are referred to as second-order cone constraints (otherwise known as Lorentz cone or ice-cream cone constraints). Thus the optimisation problem defined by Eqs. (1) to (3) can explicitly be written as a second-order cone optimisation problem as follows:

$$
\text { minimize } c^{T} x
$$

subject to:

$$
\bar{a}_{i}^{T} x+\left\|\mathrm{P}_{i} x\right\| \leq b_{i}, i=1, \ldots, m
$$

The norm term is the usual Euclidean norm and can be thought of as a penalty term which introduces some robustness within the optimisation problem.

SOCO problems can be solved efficiently through the interiorpoint methods developed recently (Andersen and Andersen, 1999; Boyd et al., 1994; Sturm, 1999). A few applications of SOCO problems have been reported in the literature. They include antenna array weight design, filter design, grasping force optimisation, portfolio optimisation, truss design, and equilibrium of systems with piecewise-linear springs design. From the literature, the reported applications are basically in the areas of electrical engineering, mechanical engineering, economics, and structural engineering (Ndambuki et al., 2000a,b; Boyd et al., 1994; Lobo et al., 1998; Ben-Tal and Nemirovsky, 1998; Boyd et al., 1998). To the best of our knowledge, this is the first time this novel tool has been applied to a real-world case aquifer.

\section{The study area}

The research was conducted within the Ewaso Ng'iro catchment situated in the central part of Kenya. This area extends from about

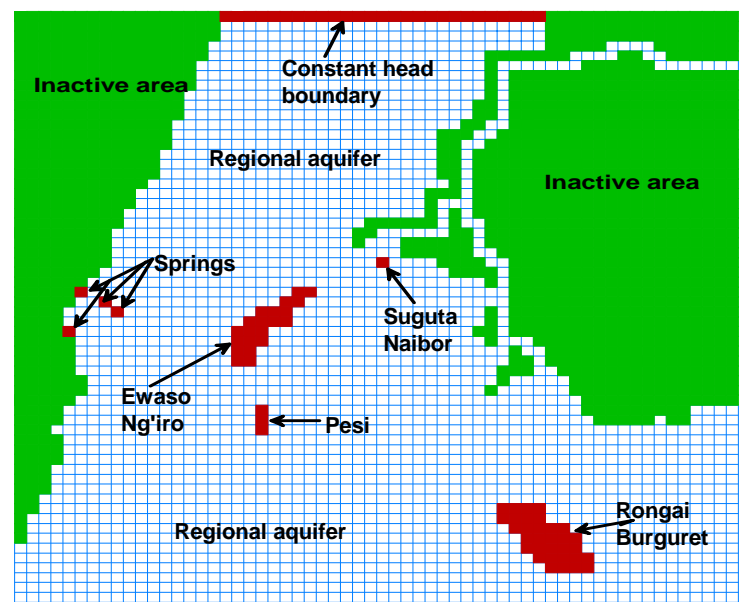

Figure 2

Modelled area

$0^{\circ} 15^{\prime}$ south of the equator to $10^{\circ} 30^{\prime}$ north and between longitudes $36^{\circ} 15^{\prime}$ east and $39^{\circ} 45^{\prime}$ east. It is the domain formed by the area of Upper Ewaso Ng'iro Basin, upstream of the confluence of the Ewaso $\mathrm{Ng}$ 'iro and Ewaso Narok Rivers and the area south-east of the confluence. This area, shown in Fig. 1 is wholly underlain by Precambrian basement rocks and is bounded to the west by the Laikipia Escarpment and to the south-east and south-west by the volcanic rocks of the Aberdares ( 3999 m) and Mount Kenya (5 $199 \mathrm{~m}$ ). The rocks from the higher parts of the study area are mainly volcanic, while in the lower areas, metamorphic rocks of the basement system predominate.

\section{Discretisation of the model}

To delineate the area of interest (regional aquifer system) from the local aquifers, the whole study area was discretised into $60 \times 60$ cells each of $1850 \mathrm{~m} \times 1850 \mathrm{~m}$. The modelled area of interest is shown in Fig. 2.

Three main rivers, i.e. Ewaso Ng'iro, Ewaso Narok and Nanyuki, were also modelled within the area of interest (Fig. 1). Further, four swamps (Ewaso Narok, Pesi, Rongai Burguret and Suguta Naibor Swamps) and four perennial springs were included in the modelling exercise as well (Fig. 2).

The overall modelling effort was intended to manage the water resources (especially groundwater) of this area in such a manner so as to maximise benefits derived without substantially imparting undesirable consequences on the ecosystem.

\section{Statement of the management problem}

Following the exposition on SOCO problems already presented, the SOCO groundwater management problem was formulated as follows:

$$
\operatorname{mimimize} \sum_{j=1}^{N_{w}} \lambda_{j} r_{j} x_{j}
$$

subject to:

$$
\begin{aligned}
& \bar{a}_{i}^{j} x+\left\|\mathrm{P}_{i} x\right\| \leq b_{i} ; i=1, \ldots, N_{c} \\
& \sum_{j \in Z_{e}} x_{j} \geq D_{l} ; l=1, \ldots, 3, Z_{e}=1, \ldots, N_{w} \\
& \sum_{j \in Z_{e}} x_{j} \geq D_{g}
\end{aligned}
$$




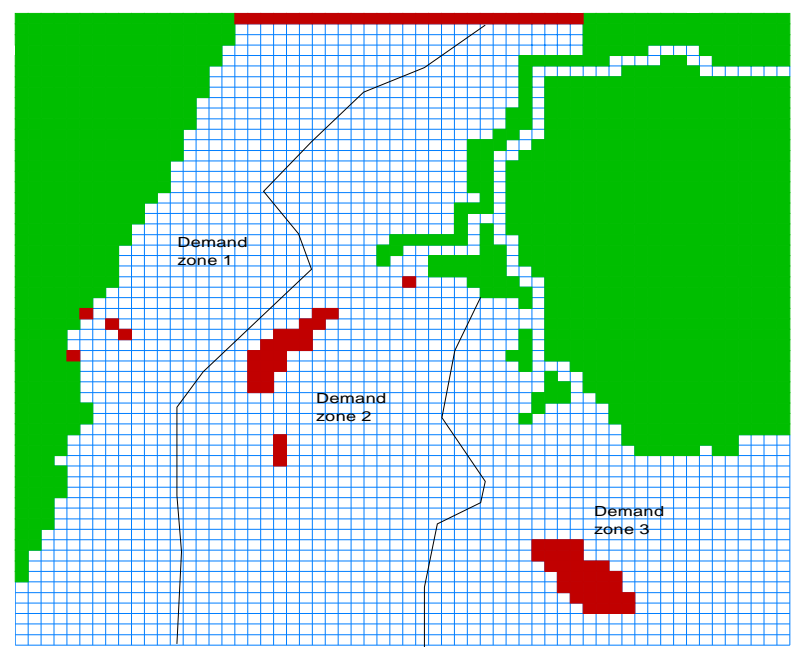

Figure 3

Local demand zones

$$
\begin{aligned}
& \sum_{j \in Z_{e}} x_{j} \leq R_{t} \\
& x_{j}^{\min } \leq x_{j} \leq x_{j}^{\max }
\end{aligned}
$$

where:

$\mathrm{N}_{\mathrm{w}}$ is the number of pumping wells

$\mathrm{N}_{c}$ is the number of control points

$\mathrm{Z}_{\mathrm{e}}^{\mathrm{c}}$ is the number of control points

$\lambda_{\mathrm{j}}$ is daily operational cost in monetary unit (MU) per unit volume per unit lift in cell $\mathrm{j}$

$\mathrm{x}_{\mathrm{j}}$ is pumping rate in cell $\mathrm{j}$

$r_{j}$ is the pumping lift in cell $j$ given by $\left(\mathrm{H}_{\mathrm{j}}-\mathrm{h}_{\mathrm{j}}\right)$

$\mathrm{H}_{\mathrm{j}}$ is height of ground surface (measured from bottom of aquifer) at cell $\mathrm{j}$

$h_{j}$ is the head in pumping cell $j$

$a_{i-}$ overbar $j$ is the mean response at control point $i$ due to pumping in cell $\mathrm{j}$

$b_{i}$ is the constraining value at control point $i$

$\mathrm{R}_{1}$ is recharge in demand zone 1

$\mathrm{x}_{\mathrm{j}}{ }^{\mathrm{min}}$ and $\mathrm{x}_{\mathrm{j}}{ }^{\mathrm{max}}$ are the minimum and maximum allowable pumping rates;

$\mathrm{D}$ is total water demand

$l \stackrel{g}{\text { refers }}$ to different water demand zones as shown in Fig. 3 .

\section{Solution methodology}

To solve the above formulated robust single objective SOCO groundwater quantity problem, the following steps were followed:

- A number of realisations, w, of the uncertain parameter (in our case, the aquifer transmissivity field) were generated. Each realisation,w, has different spatial values of transmissivity. This means that every realisation, $w$, gives rise to different responses $a_{i}$ at locations of interest and hence different values of mean $a_{i}$,s and $\mathbf{P}_{i}$.

- Using the groundwater simulation software, MODFLOW (McDonald and Harbaugh, 1984) and the generated transmissivity fields, the groundwater aquifer responses, $a_{i}$, at all points of interest due to unit pumping rates at all candidate well locations were determined.

- The nominal values of $a_{i}$ and the perturbations, $\mathbf{P}_{i}$ were computed.

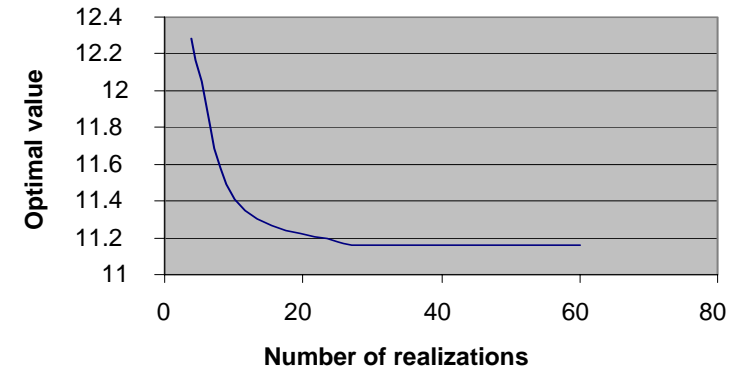

Figure 4

Variation of optimal value with number of Monte Carlo

\begin{tabular}{|c|c|c|}
\hline \multicolumn{3}{|c|}{$\begin{array}{c}\text { TABLE } 1 \\
\text { Parameters used to generate } \\
\text { transmissivity fields }\end{array}$} \\
\hline & $\begin{array}{c}\text { Standard } \\
\text { deviation } \\
(\log 10)\end{array}$ & $\begin{array}{l}\text { Correlation } \\
\text { length/ } \\
\text { field width }\end{array}$ \\
\hline Case 1 & 0.5 & 0.5 \\
\hline Case 2 & 1.0 & 0.5 \\
\hline Case 3 & 1.5 & 0.5 \\
\hline
\end{tabular}
realisations

- Using the single objective optimisation software, SeDuMi (Sturm, 1999), the single objective SOCO problem was solved.

\section{Input data}

The following data were used in this research:

Recharge $24.7 \times 10^{3} \mathrm{~m}^{3} \cdot \mathrm{d}^{-1}$

Mean (harmonic) transmissivity $6.073 \mathrm{~m}^{2} \cdot \mathrm{d}^{-1}$

Current amount of water supplied $6.2 \times 10^{3} \mathrm{~m}^{3} \cdot \mathrm{d}^{-1}$

Projected global (year 2000) water demand $11.1 \times 10^{3} \mathrm{~m}^{3} \cdot \mathrm{d}^{-1}$

Projected Zone 1 water demand $2.07 \times 10^{3} \mathrm{~m}^{3} \cdot \mathrm{d}^{-1}$

Projected Zone 2 water demand $5.13 \times 10^{3} \mathrm{~m}^{3} \cdot \mathrm{d}^{-1}$

Projected Zone 3 water demand $3.86 \times 10^{3} \mathrm{~m}^{3} \cdot \mathrm{d}^{-1}$.

\section{Results}

The grid size used is big, hence the results should be viewed within that context. To arrive at a reasonable number of Monte Carlo realisations to be used in the estimation of the uncertainty ellipsoids, we carried out an investigation to determine how the optimal objective value changes as the number of Monte Carlo realisations were increased. Figure 4 shows how the optimal objective value changes with the number, $\Omega$, of Monte Carlo realisations considered. It shows that the optimal objective value changes widely for number of realisations ranging between 4 and 30. However, for more than 30 Monte Carlo realisations, the optimal value tends to stabilise. Thus, it was felt that consideration of 30 Monte Carlo realisations was a good compromise between $\mathrm{CPU}$ time and solution accuracy.

The results reported in this paper, therefore, were based on 30 realisations of transmissivity fields. These fields were generated using a mean (harmonic) transmissivity $(\log 10)$ of 0.7834 and other parameters shown in Table 1. In total, three cases with the parameters shown in Table 1 were analysed.

As is evident from Table 2 and Fig. 4 increasing the value of the 


\begin{tabular}{|l|c|}
\hline \multicolumn{2}{|c|}{ TABLE 2 } \\
Optimal objective values for \\
the cases shown in Table 1
\end{tabular}

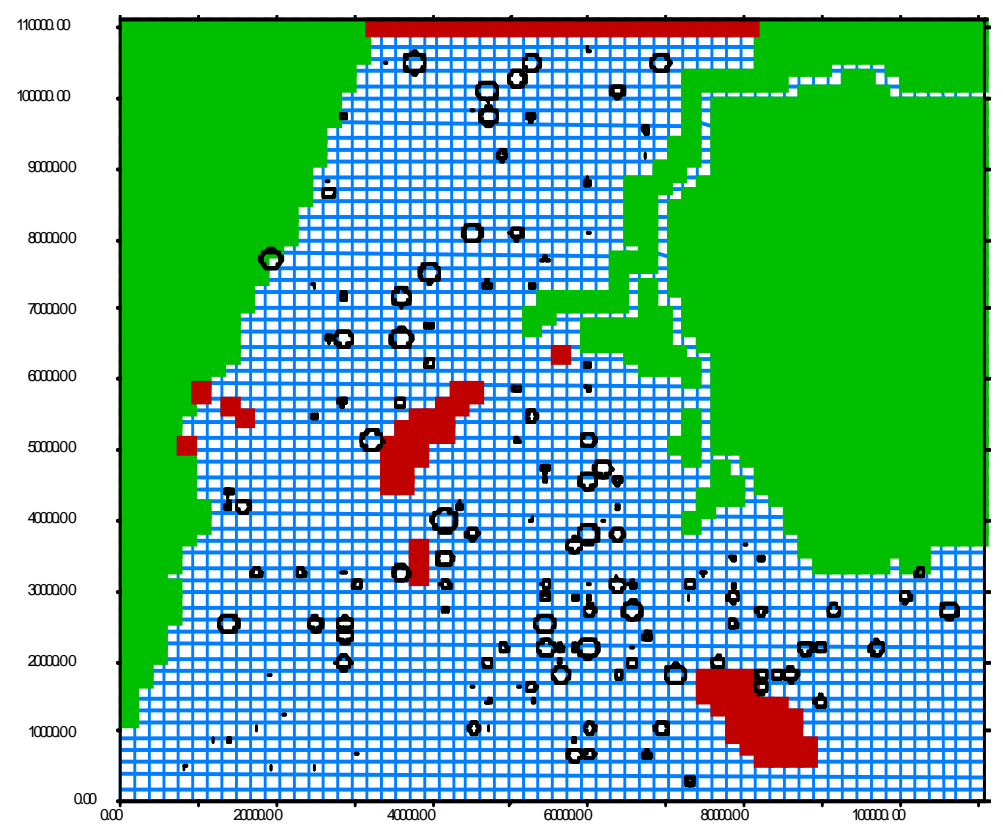

Figure 5

Un-optimised strategy

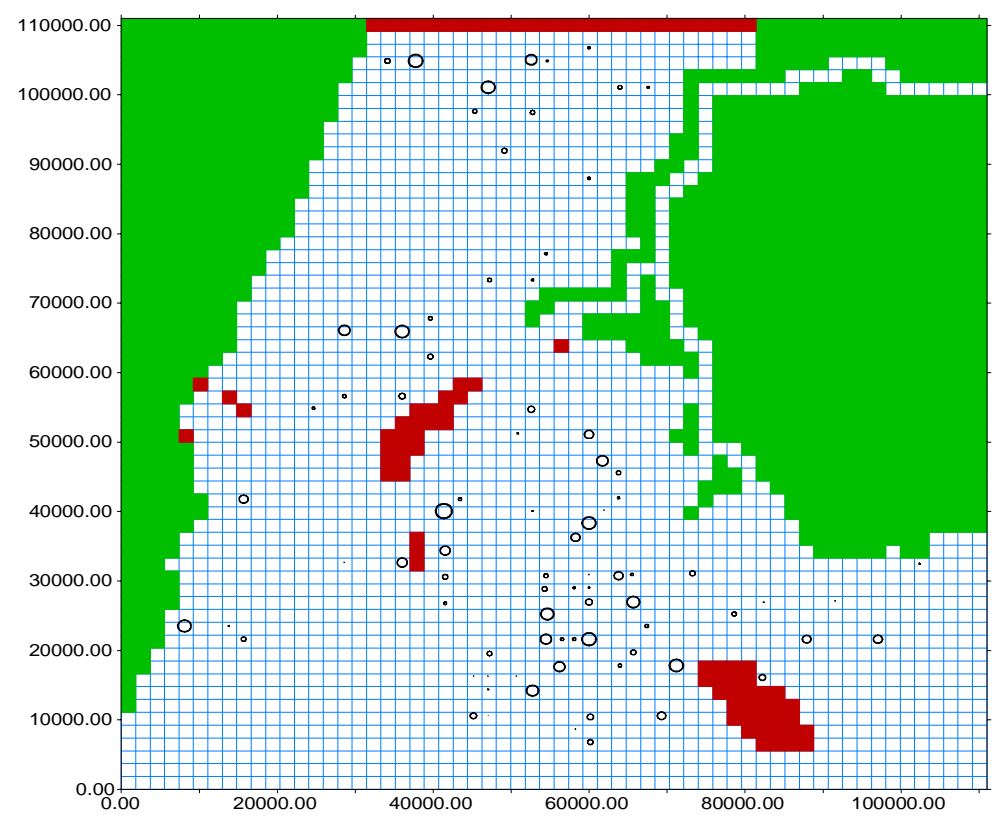

Figure 6

Optimised strategy corresponding to Case 1 of Table 1 standard deviation results in an increase in the cost of the optimal solution as expected. This is because increasing the value of standard deviation is equivalent to increasing the volume of the uncertainty ellipsoids. Thus an optimal solution sought when considering high magnitudes of uncertainty is likely to be more costly since such optimal solutions will have to be robust against higher levels of uncertainty.

Figure 5 shows the present un-optimised pumping strategy, able to supply a total water demand of $6.2 \times 10^{3} \mathrm{~m}^{3} \cdot \mathrm{d}^{-1}$ (which is about $50 \%$ of the required supply), while Fig. 6 shows the realised optimal pumping solution. The realised optimal pumping strategy recommends the mobilisation of a total of 140 pumping wells stressing the aquifer at rates higher than the minimum permissible rates. Thus for the groundwater aquifer to satisfy the projected water demand of $11.1 \times 10^{3} \mathrm{~m}^{3} \cdot \mathrm{d}^{-1}$, an additional 56 boreholes will have to be rejuvenated.

Comparison of Fig. 5 with Fig. 6 shows that the optimised strategy has phased out some of the active pumping wells within the present un-optimised strategy, an indication that such pumping wells were not optimally placed during the initial phases of aquifer exploitation.

\section{Conlusions}

Because it is virtually impossible to resolve the uncertainty in groundwater aquifer material properties (transmissivity) to such a detail, which would quantify the use of deterministic optimisation approaches, water managers need robust solutions which do not change with small changes in the input transmissivity values. In this paper, we have shown how groundwater quantity optimisation problems whose transmissivity values are uncertain can conveniently be reformulated as second-order cone optimisation problems, which are amenable to solution through interior-point methods. Moreover, we have proposed a solution methodology to such problems and its suitability is clearly shown through a real-world case optimisation problem.

An outstanding feature of this approach is that one is able to circumvent the shortfalls of the popular Monte Carlo approach through consideration of only a few realisations of the uncertain transmissivity field in order to estimate the sizes and shapes of the uncertainty ellipsoids. This leads to significant saving in terms of CPU time, hence allowing optimisation problems of high dimensions to be solved within a relatively shorter time. An extra added advantage (though not discussed in this paper) is the versatility of the approach. This is borne out by the ease with which one can increase or decrease the robustness of the optimal solution by either scaling up or scaling down the uncertainty ellipsoids at very little extra effort in terms of CPU time.

\section{References}

ANDERSEN ED and ANDERSEN KD (1999) Exploiting parallel hardware to solve optimization problems. SIAM News 32 (4). 
BEN-TAL A and NEMIROVSKI A (1998) Convex Optimization in Engineering. Technion-Israel Institute of Technology.

BOYD S, CRUSIUS G and HANSSON A (1998) Control applications of non-linear convex programming. J. Process Control 8 (5-6) 313-324.

BOYD S, VANDENBERGHE L and GRANT M (1994) Efficient convex optimization for engineering design. In: Proc. IFAC Symposium on Robust Control Design. Rio de Janeiro, Brazil.

LOBO MS, VANDENBERGHE L, BOYD S and LEBRET H (1998) Applications of second-order cone programming. Linear Algebra and its Applications 284 193-228.

MARK W, MORTON DP and WOOD RK (1999) Monte Carlo bounding techniques for determining solution quality in stochastic programs. Oper. Res. Lett. 24 47-56.

MCDONALD MG and HARBAUGH AW (1994) A Modular ThreeDimensional Finite Difference Ground-Water Flow Model. Scientific Publications Co.
MULVEY JM, VANDERBEI RJ, ZENIOS SA (1995) Robust optimization of large-scale systems. Oper. Res. 43 (2) 264-281.

NDAMBUKI JM, OTIENO FAO, STOET CBM and VELING EJM (2000a) Groundwater management under uncertainty: A multi-objective approach. Water SA 26 (1) 35-42.

NDAMBUKI JM, STOET CBM, VELING EJM and TERLAKY T (2000b) Robust groundwater management through second order cone programming (SOCP). In: Oliver Sililo et al. (eds.) Groundwater: Past Achievements and Future Challenges. 413-417.

STURM JF (1999) Using SeDuMi 1.02, A Matlab Toolbox for Optimization over Symmetric Cones. In: Potra F, Roos C, Terlaky T (eds.) Optimization Methods and Software. Vol. 11-12 Special Issue on Interior Point Methods. 625-654.

WAGNER JM, SHAMIR U and NEMATI HR (1992) Groundwater quality management under uncertainty: Stochastic programming approaches and the value of information. Water Resour. Res. 28 (5) 1233-1246. 
